\title{
Análise comparativa da colheita florestal em regime de manejo de alto fuste e talhadia
}

\author{
Estevão Giacomin ALVES $^{1}$, Nilton Cesar FIEDLER ${ }^{1 *}$, Elzimar Oliveira GONÇALVES ${ }^{1}$, \\ Leandro Christo BERUDE ${ }^{1}$
}

\author{
${ }^{1}$ Departamento de Ciências Florestais e da Madeira, Universidade Federal do Espírito Santo, Jerônimo Monteiro, ES, Brasil. \\ *E-mail: fiedler@pq.cnpq.br
}

Recebido em março/2017; Aceito em janeiro/2018.

\begin{abstract}
RESUMO: No setor florestal é muito comum a discussão que a colheita em regime de manejo de talhadia proporciona menor produtividade das máquinas que no regime de alto fuste. Porém, os trabalhos na literatura são muito escassos para comprovar tal afirmação. Objetivou-se no presente trabalho realizar uma análise comparativa da colheita florestal mecanizada em área de regime de manejo de alto fuste e talhadia, analisando os parâmetros técnicos do harvester (colhedor florestal). A pesquisa foi realizada em áreas de colheita de eucalipto, no município de Aracruz, Espírito Santo. Foram avaliados dois tratamentos, sendo eles: talhadia e alto fuste. Utilizou-se quatro máquinas do mesmo modelo, com os mesmos operadores, durante quatro dias de trabalho em cada tratamento, sempre no primeiro turno, totalizando 16 repetições em cada tratamento. A comparação das médias foi feita utilizando o teste $\mathrm{t}$, em nível de $95 \%$ de probabilidade. Não observou-se diferença significativa entre a produtividade da máquina em regime de manejo de talhadia e alto fuste. Entretanto, o número de quedas de corrente para a colheita em talhadia foi 1,59 vezes maior e estatisticamente significativo.
\end{abstract}

Palavras-chave: rebrota, corte florestal, reforma, eucalipto.

\section{Comparative analysis of forest harvesting of tall trees regime and coppice}

\begin{abstract}
In forestry companies, it is common the discussion that in coppice harvesting occurs losses in machine productivity, although the works in the literature are very scarce to prove such a claim. The objective of this work was to perform a comparative analysis of mechanized forest harvesting in the area of tall trees and coppice management regime, analyzing the technical parameters of the forest harvester. The research was conducted in eucalyptus cutting areas in the county of Aracruz, Espirito Santo. Two treatments were evaluated, namely: coppice and tall trees. Four machines of the same model were used, with the same operators, for four working days in each treatment, always in the first shift, in a total of 16 repetitions for each treatment. The comparison of means was performed using the $t$ test, at a $5 \%$ level of probability. No difference was observed between the productivity of the machine in coppice management regime and tall trees, however, the number of chain falls for coppice harvesting was statistically significant (1.59 times higher).
\end{abstract}

Keywords: regrowth, forestry, reforestation, eucalyptus.

\section{INTRODUÇÃO}

Os decorrentes acontecimentos nos cenários político e econômico brasileiro provocaram uma retração em praticamente todos os setores econômicos do Brasil no ano de 2016; no entanto, o setor florestal foi um dos menos afetados, uma vez que apresentou queda apenas de 3,3\% no PIB. No mesmo período o setor de árvores plantadas do país e o setor de celulose aumentaram sua produção e exportação, elevando o Brasil para o $2^{\circ}$ lugar no ranking de produção mundial. Outros setores florestais que também atingiram grandes marcas no último ano; foram o setor de madeira serrada que exportou $39 \%$ a mais em relação a 2015 , e a produção de painéis compensados a partir de árvores plantadas aumentou 3,8\%. (IBÁ, 2017). Grande parte da importância do setor se deve às espécies do gênero Eucalyptus, que se consolidaram como importante fonte de suprimento de madeira por apresentarem um ciclo de corte curto e rápido crescimento, representando $71,9 \%$ das áreas de florestas plantadas, totalizando cerca de 5,56 milhões de hectares no País.
A perpetuação dos povoamentos florestais de eucalipto pode ser feita por meio de três regimes: alto fuste, talhadia simples e talhadia composta.

No sistema de alto fuste, também conhecido como corte/reforma, após o corte raso da floresta, realiza-se o replantio da área (reforma) que normalmente é realizado com a troca de material genético na entrelinha do plantio antigo. No manejo do alto fuste são necessários tratos culturais à formação da floresta (preparo do solo, plantio, irrigação, adubação, controle de pragas, doenças e da matocompetição).

O sistema silvicultural de talhadia simples se caracteriza por ser aquele no qual, após o corte das árvores existentes numa floresta, as gemas dormentes ou adventícias dos tocos e, ou raízes que permaneceram na área se desenvolvem, emitindo brotações que iniciam um novo ciclo florestal, sendo, portanto aplicável apenas às espécies florestais que tenham capacidade de brotar após o corte raso.

A diferença entre talhadia simples e composta, é que na talhadia simples todo o povoamento é proveniente das brotações que foram emitidas dos tocos do plantio anterior. Já 
na talhadia composta há uma conjugação dos regimes anteriores (alto-fuste e talhadia simples) no mesmo povoamento. Portanto, é uma composição mista dos dois regimes anteriores.

Na década de 60 até o início da década de 80 , a opção do uso da talhadia era definida como sistema padrão. No entanto, devido às inúmeras espécies/procedências de Eucalyptus utilizadas, à diversidade de sítios edafoclimáticos implantados e aos diferentes níveis tecnológicos de implantação e colheita adotados, a produtividade florestal obtida na segunda rotação mostrou-se extremamente variável, comparativamente àquela obtida na primeira rotação.

Essa preferência pelo sistema de alto fuste iniciada na década de 80 , foi fortemente motivada pela adequação de material genético, onde foram substituídas espécies/procedências de menores produtividades por materiais melhorados, com maiores produtividades e mais adaptados às condições de sítio (STAPE, 1997).

Recentemente, tem crescido o interesse de manejo por talhadia dos povoamentos de eucalipto de maior produtividade, uma vez que apresenta vantagens como: uma alta taxa de crescimento inicial, maior proteção do solo, redução dos custos de produção e vantagens operacionais pois com a diminuição das operações, diminui também a necessidade de mão de obra, facilitando para produtores e empresas a regeneração de suas áreas.

São escassas as pesquisas que avaliam, do ponto de vista da colheita florestal, qual o melhor sistema de regeneração. Nas empresas florestais é comum a discussão de que a colheita de talhadia, mesmo quando possui apenas um fuste por cepa é menos viável economicamente, chegando à perda de produção de até $10 \%$ no momento do corte. Outra questão é o fato de um maior número de quedas de corrente do sabre do colhedor florestal harvester.

A colheita florestal é atividade complexa, dado o grande número de variáveis que afetam a produtividade e, consequentemente, os custos operacionais (CANTO et al., 2006), representando cerca de $50 \%$ do custo total da madeira posta na indústria (MACHADO, 2014). A partir disso, se faz necessária uma análise operacional da colheita florestal em regime de alto fuste comparando-as com o regime de talhadia.

Objetivou-se com essa pesquisa analisar tecnicamente e comparativamente as atividades operacionais na colheita florestal de Eucalyptus spp sob regime de manejo de alto fuste e talhadia.

\section{MATERIAL E MÉTODOS}

\subsection{Caracterizações da área de estudo}

A pesquisa foi realizada em plantios clonais de híbridos de Eucalyptus urophylla $x$ Eucalyptus grandis em espaçamento de $3 \times 3$ metros e idade média de cinco anos, localizadas no município de Aracruz, estado do Espírito Santo. Todos os talhões avaliados estavam em relevos caracterizados como planos.

\subsection{Coleta de dados}

A análise foi realizada com colhedor florestal (harvester) da marca Komatsu, modelo PC200, com cabeçote Harvester Komatsu 370E. Foram selecionadas quatro máquinas, juntamente com seus respectivos operadores, onde avaliou-se sempre os mesmos conjuntos máquina/operador em ambos os tratamentos. Os dados foram coletados em quatro dias em cada máquina, sempre no mesmo talhão e no primeiro turno, totalizando assim, dezesseis repetições por tratamento.

A seleção dos talhões foi realizada de acordo com um inventário pré-corte, avaliando o volume médio individual (VMI) iguais em ambos talhões utilizados na coleta. Avaliouse também a homogeneidade em todo talhão, com proximidade produtiva de uma planta para outra, e também se não possuíam concentrações de falhas ou áreas que passaram por algum distúrbio durante o plantio, como ataque de pragas, doenças, alagamentos, incêndios.

O VMI das árvores avaliadas para o talhão de regime de manejo de alto fuste e talhadia foi de $0,12 \mathrm{~m}^{3}$ sem casca.

Foi realizado roçada manual na brotação lateral, também conhecidos como "broto ladrão", comumente encontrado em manejo de talhadia. Sendo assim, como no manejo de alto fuste não possui tais brotações, realizou-se a roçada a fim de padronizar a não existência destas brotações em ambos tratamentos.

\subsection{Operações e fases estudadas}

Coletou-se os dados por meio de um estudo de tempos e movimentos, caracterizado pelo método de tempos contínuos com uso de cronômetro digital e formulário para registro dos dados. O acompanhamento das operações e fases foi in loco, conforme o andamento normal das atividades na empresa, sem qualquer interferência. Dividiu-se os tempos gastos durante a jornada de trabalho em cinco categorias, conforme proposto por Lacerda (2015).

(1) Tempo Acessório: Funções obrigatórias, porém não diretamente relacionadas com a operação, como paradas pessoais, paradas por decisões gerenciais;

(2) Tempo Auxiliar: Funções que obrigatoriamente são exigidas pela operação, sem as quais essa não ocorreria (abastecimento, lavagem);

(3) Tempo Improdutivo: Máquina está disponível para a operação, porém não está sendo utilizada (aguardando peças e ou mecânicos);

(4) Tempo em Manutenção: Manutenção preventiva ou corretiva da máquina (considerado o tempo gasto para manutenção da máquina base e implementos);

(5) Tempo Produtivo: Operação efetiva de colheita florestal (abate, processamento e embandeiramento).

\subsection{Estudos de tempos e movimentos}

O ciclo operacional foi subdividido nas seguintes fases:

(a) Deslocamento da Máquina: movimentação da máquina em direção à próxima árvore a ser derrubada, se encerrando com o começo da movimentação do cabeçote.

(b) Movimentação do cabeçote: movimentação somente do cabeçote em direção à próxima árvore, até o encaixe na árvore.

(c) Abate: tempo gasto após o fechamento das garras do cabeçote na árvore até o momento da derrubada completa da mesma.

(d) Processamento: ações de desgalhar a árvore, descascar e seccionar a árvore em toretes, bem como a ação de simples passada do rolo pelo cabeçote.

(e) Descarte e apoio para pilha: consistiu no uso do cabeçote para retirada de resíduos do corte e para realizar o "travesseiro" ou suporte para pilha. 
(f) Recuperação: consistiu em casos em que o operador derrubava várias árvores e depois as processava, ou quando por alguma razão soltava a árvore após a derrubada.

(g) Interrupções: fatores operacionais ou não operacionais diversos como parada para alimentação, hidratação, manutenção corretiva ou preventiva, abastecimento da máquina, chuva e necessidades pessoais.

\subsection{Disponibilidade mecânica}

A disponibilidade mecânica refere-se ao percentual da jornada de trabalho em que a máquina se encontra mecanicamente apta para execução de suas funções, ou seja, baseia-se em um horizonte planejado desconsiderando o tempo em que ela está em manutenção (equação 1).

$$
\mathrm{DM}=[(\mathrm{HT}-\mathrm{HM}) / \mathrm{HT}] * 100 \quad \text { (Equação 1) }
$$

em que: $\mathrm{DM}=$ disponibilidade mecânica (\%); HT = tempo total de trabalho $(\mathrm{h}) ; \mathrm{HM}=$ tempo em manutenção $(\mathrm{h})$.

\subsection{Grau de utilização}

Considerou-se como a porcentagem do tempo efetivamente trabalhado pela máquina (equação 2):

$$
\mathrm{GU}=[\text { he } /(\text { he }+\mathrm{hp})] * 100
$$

em que: GU = Grau de utilização (\%); he = Horas efetivas de trabalho (h); hp = Horas paradas operacionais (h).

\subsection{Eficiência Operacional}

A eficiência operacional expressa a percentagem do tempo efetivamente trabalhado. Refere-se à execução das atividades em relação ao total programado para o trabalho (VASCONCELLOS et al., 2006) (equação 3).

$$
\mathrm{EOp}=(\mathrm{Tef} / \mathrm{T}) * 100
$$

(Equação 3)

em que: $\mathrm{EOp}=$ eficiência operacional $(\%)$; Tef $=$ Tempo efetivo (h); $\mathrm{T}=$ tempo total $(\mathrm{h})$.

\subsection{Produtividade operacional}

A produtividade expressa uma razão entre entre o volume e o tempo efetivo de trabalho (he), o qual é representado pelo número total de horas descontando-se as interrupções mecânicas ou operacionais quando ocorrerem (equação 4).

$$
\mathrm{P}=(\mathrm{n} * \mathrm{VMI}) / \text { Tef }
$$

(Equação 4)

em que: $\mathrm{P}=$ produtividade $\left(\mathrm{m}^{3} \cdot\right.$ he- 1$) ; \mathrm{n}=$ número de árvores cortadas (un); VMI = volume médio individual $\left(\mathrm{m}^{3}\right)$; Tef = tempo efetivo de trabalho (he).

2.9. Número de horas efetivamente operadas por queda de corrente

O número de horas efetivamente operadas por queda de corrente é expressa pela equação 5:

$$
\text { Hef } / \mathrm{qc}=\mathrm{Hef} / \mathrm{QC}
$$

(Equação 5)

em que: Hef/qc = número de horas efetivamente operada por queda de corrente (h.un-1); Hef = somatório de horas efetivamente operada $(\mathrm{h}) ; \mathrm{QC}=$ somatório de queda de corrente (un).
2.10. Número de árvores cortadas por queda de corrente do cabeçote

O número de árvores cortadas por queda de corrente é expressa pela equação 6 .

$$
\mathrm{ac} / \mathrm{qc}=\mathrm{AC} / \mathrm{QC}
$$

em que: ac/qc $=$ número de árvores cortadas por queda de corrente; $\mathrm{AC}=$ somatório de árvores cortadas; $\mathrm{QC}=$ somatório de queda de corrente.

\subsection{Procedimento estatístico}

Foram observadas as pressuposições do teste de normalidade pelo teste de Shapiro-Wilk e de homogeneidade de variância dos dados pelo teste de Barlett referentes às características avaliadas. A comparação de médias foi feita utilizando-se o teste $\mathrm{t}$, em nível de $5 \%$ de probabilidade, utilizando o pacote ExpDes do software R 2.13.1 (Ferreira et al., 2011).

\section{RESULTADOS}

\subsection{Operações e fases estudadas}

A caracterização dos tempos operacionais durante a coleta de dados para os tratamentos é ilustrada na Figura 1.

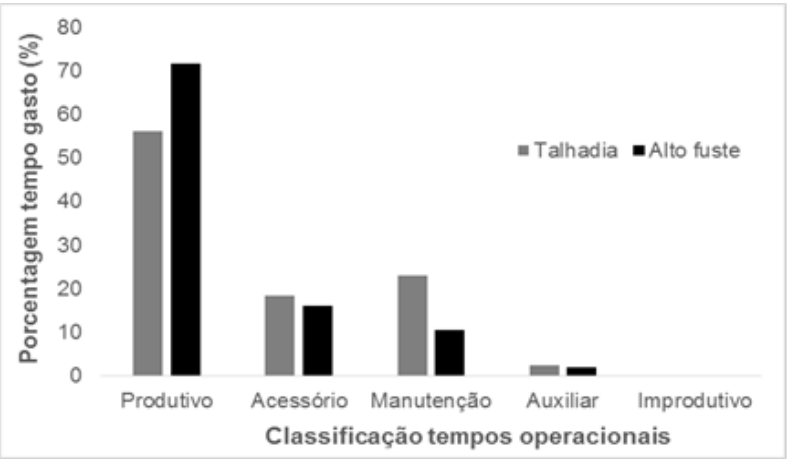

Figura 1. Caracterização dos tempos operacionais para talhadia e alto fuste.

Figure 1. Characterization of the operating times for coppice and tall trees.

\subsection{Estudos de tempos e movimentos no corte}

Os tempos gastos no ciclo operacional de corte $\mathrm{e}$ processamento das árvores para os regimes de talhadia e alto fuste são ilustrados na Figura 2.

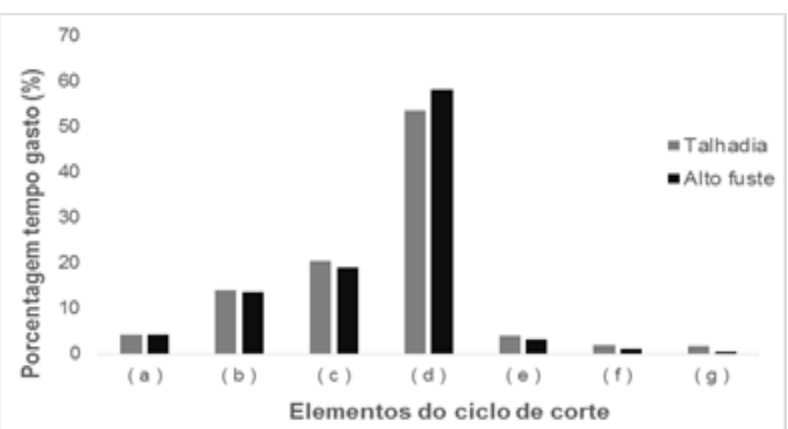

Nota: (a) deslocamento da máquina; (b) posicionamento do cabeçote; (c) derrubada; (d) processamento; (e) recuperação; (f) descarte e apoio para pilha; (g) interrupções.

Figura 2. Percentuais de tempo gasto em cada elemento do ciclo operacional para talhadia e alto fuste.

Figure 2. Percentage of time spent in each operating cycle of the element to coppice and tall trees. 


\subsection{Fatores operacionais}

A distribuição dos parâmetros de disponibilidade mecânica (DM), grau de utilização (GU) e eficiência operacional (Eop) para os regimes de talhadia e alto fuste podem ser observadas na Figura 3.

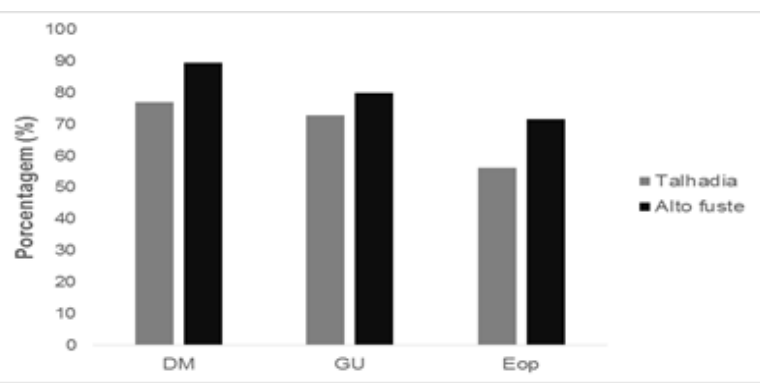

Figura 3.Disponibilidade mecânica, grau de utilização e eficiência operacional para talhadia e alto fuste.

Figure 3. Mechanical availability, degree of utilization and operational efficiency for coppice and tall trees.

3.4. Análise produtiva das máquinas entre os regimes de talhadia e alto fuste

Os dados de produtividade das máquinas durante a pesquisa, para os tratamentos de talhadia e alto fuste, são ilustrados na Figura 4

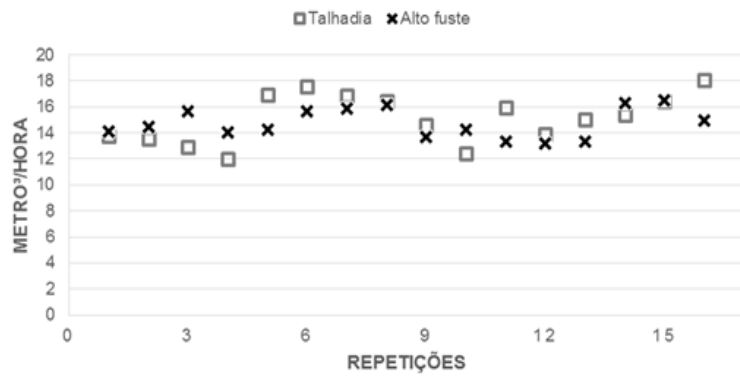

Figura 4. Produtividade das máquinas $\left(\mathrm{m}^{3} \mathrm{~h}^{-1}\right)$ para talhadia e alto fuste.

Figure 4. Machine productivity $\left(\mathrm{m}^{3} \mathrm{~h}^{-1}\right)$ for coppice and tall trees.

Os valores médios de produtividade, número de árvores cortadas para cada queda de corrente e número de horas trabalhadas por queda de corrente podem ser observados na Tabela 1.

Tabela 1. Médias de produtividade, horas efetivas por queda de corrente (Hef/qc) e número de árvores por queda de corrente (ac/qc) observadas para talhadia e alto fuste.

Table 1. Productivity averages, actual hours by falling chain (Hef/qc) and number of trees per chain falls (ac/qc) observed for coppice and tall trees.

\begin{tabular}{cccc}
\hline & Produtividade $\left(\mathrm{m}^{3} \mathrm{~h}^{-1}\right)$ & Hef/qc & $\mathrm{ac} / \mathrm{qc}$ \\
\hline Talhadia & $15,12 \mathrm{a}$ & $10,51 \mathrm{~b}$ & $1335 \mathrm{~b}$ \\
Alto fuste & $14,75 \mathrm{a}$ & $16,97 \mathrm{a}$ & $2119 \mathrm{a}$ \\
\hline
\end{tabular}

Nota: Médias seguidas pela mesma letra na coluna não diferem entre si, pelo teste $\mathrm{t}$ a $5 \%$ de probabilidade.

\section{DISCUSSÃO}

Como era esperado, o maior tempo foi classificado como produtivo, porém nota-se que o tratamento que avaliou a colheita em regime de talhadia apresentou valores menores quando comparados com o regime de alto fuste. $\mathrm{O}$ fato de ocorrer menor tempo produtivo para o tratamento em regime de talhadia não é relacionado ao tratamento em si, e sim ao fato de um maior número de manutenções. Tais manutenções não decorrem do fato intrínseco ao regime em si e sim ao fato das máquinas avaliadas terem certo grau de uso, onde ocorreu a necessidade de manutenções corretivas diversas durante o desenvolvimento da pesquisa.

Não foi observado tempo improdutivo durante a classificação dos tempos operacionais, uma vez que os operadores têm alto grau de treinamento, direcionado também para evitar perdas de tempo, aliado à logística operacional bem estruturada por parte da empresa, tanto dos operadores, como mecânicos e peças de reposição.

Avaliando os tempos e movimentos da máquina no momento do corte/processamento das árvores, como era esperado, notou-se um maior tempo gasto para o processamento (descascamento, desgalhamento, traçamento e destopamento), apresentando valores próximos à $60 \% \mathrm{em}$ ambos regimes de manejo, seguidos de abate e movimentação do cabeçote.

Silva et al. (2014), encontrou para dois diferentes modelos de harvester, média de $53,88 \%$ e $54,84 \%$ para o processamento, respectivamente, sendo valores considerados similares aos encontrados neste trabalho.

O aumento do tempo gasto com manutenção, é inversamente proporcional a disponibilidade mecânica da máquina. Logo, o regime de talhadia apresentou menores taxas quando comparado com o regime de alto fuste. Além de ter apresentado menor disponibilidade mecânica, também apresentou menor grau de utilização $(72,93 \%)$, ou seja, mesmo quando a máquina estava disponível para ser utilizada, a mesma teve menor uso quando comparado ao avaliado no regime de alto fuste, consequentemente, apresenta assim também menor eficiência operacional $(56,20 \%)$, enquanto o regime de alto fuste apresentou valores superiores a $70 \%$.

De acordo com Machado (2014), uma recomendação é feita em que a linha ótima de aceitação para as variáveis DM, EO e GU sejam sempre iguais ou superiores à $70 \%$. Sendo assim, a eficiência operacional para o tratamento em regime de talhadia não foi satisfatória estando abaixo do recomendado.

Como pode ser observado na Figura 4, não houve predomínio de um tratamento quando comparado ao outro com relação às produtividades de colheita de madeira em metros cúbicos por hora efetivamente trabalhada.

Não foi observada diferença significativa na produtividade das máquinas (Tabela 1), pelo teste t a $5 \%$ de probabilidade. Logo, a quantidade de metros cúbicos de madeira cortada e processada por hora efetiva é igual nos diferentes regimes de manejo. Porém, o número de quedas de corrente do sabre do harvester diferiu estatisticamente, em que, em média, operou-se 10,51 horas para cada queda de corrente no regime de talhadia e 16,97 horas para uma queda de corrente no regime de alto fuste.

Avaliando o número de árvores cortadas por queda de corrente, também se encontra diferenças estatísticas entre os tratamentos. No regime de talhadia ocorreu uma queda de corrente a cada 1335 árvores cortadas e processadas. Já no regime de alto fuste, ocorreu uma queda a cada 2119 árvores cortadas e processadas.

Essa diferença entre os tratamentos pode ser explicada devido ao fato da roçada pré-corte ser realizada nas brotações laterais de forma manual, com utilização de ferramentas 
como a foice ou o facão. Observou-se em algumas cepas que o corte dessas brotações laterais foram feitos acima dos 10 centímetros de altura, ou seja, acima da altura que o sabre do harvester faz a derrubada da árvore. Assim, o toco da brotação lateral, em alguns casos, mesmo com a roçada manual continuou presente, aumentando a probabilidade de queda de corrente.

\section{CONCLUSÕES}

As distribuições dos tempos operacionais estão dentro da normalidade, com a maior parte do tempo gasto com o processamento, seguido do abate e movimentação do cabeçote, respectivamente.

A disponibilidade mecânica e o grau de utilização estão de acordo com o recomendado, com exceção da eficiência operacional para o regime de talhadia.

Não foi observado diferença signicativa na produtividade das máquinas para a colheita em regime de alto fuste e talhadia, porém constatou-se diferença significativa entre o número de árvores cortadas por quedas de corrente, sendo maior 1,59 vezes para regime de talhadia durante a operação.

\section{REFERÊNCIAS}

CANTO, J. L.; MACHADO, C. C.; GONTIJO, F. M.; JACOVINE, L. A. G. Colheita e transporte florestal em propriedades rurais fomentadas no estado do Espírito Santo. Revista Árvore, Viçosa, v. 30, n. 6, p. 989-998, 2006.

FERREIRA, E. B.; CAVALCANTI, P. P.; NOGUEIRA, D. A. Experimental Designs: um pacote $\mathrm{R}$ para análise de experimentos. Revista de Estatística da UFOP, Ouro preto - MG, p. 1-9, 2011
INDÚSTRIA BRASILEIRA DE ÁRVORES - IBÁ. Relatório IBÁ 2017. Brasília, 2017. 64 p. Disponível em: $<\quad$ http://www.iba.org/images/shared/iba_2017.pdf>. Acesso em: 26 nov. 2017.

LACERDA, L. C.; FIEDLER, N. C.; CHICHORRO, J. F.; CARMO, F. C. A.; MENDONÇA, A. R. Análise operacional da extração florestal mecanizada em módulos próprios e terceirizados. Nativa, Sinop, v. 3, n. 3, p. $205-$ 209, 2015. DOI: http://dx.doi.org/10.14583/23187670.v03n03a10

MACHADO, C. C. Colheita Florestal. 3. ed. Viçosa: Ed. UFV, 2014. 543p.

MACHADO, C. C.; LOPES, E. S. Análise da influência do comprimento de toras de eucalipto na produtividade e custo da colheita e transporte florestal. Revista Cerne, Lavras, v. 6, n. 2, p. 124-129, 2000.

SILVA, E. N.; MACHADO, C. C.; FIEDLER, N. C.; FERNANDES, H. C.; PAULA, M. O.; CARMO, F. C. A.; MOREIRA, G. R.; COELHO, F. E. Avaliação de custos de dois modelos de harvester no corte de eucalipto. Ciência Florestal, Santa Maria, v. 24, p. 741-748, 2014. DOI: http://dx.doi.org/10.5902/1980509815733

STAPE, J. L. Planejamento global e normatização de procedimentos operacionais da talhadia simples em Eucalyptus. Série Técnica IPEF, Piracicaba, v. 11, n. 30, p. 51-62, 1997.

VASCONCELLOS, V. A.; CANEN, A. G.; LINS, M. P. E. Identificando as melhores práticas operacionais através da associação Benchmarking-Dea: o caso das refinarias de petróleo. Revista Brasileira de Pesquisa Operacional, Rio de Janeiro, v. 26, n. 2, p.51-67, 2006. 